\title{
Nanofocusing of light into semiconducting fin photonic crystals
}

J. Bogdanowicz, T. Nuytten, A. Gawlik, A. Schulze, I. De Wolf, and W. Vandervorst

Citation: Appl. Phys. Lett. 108, 083106 (2016);

View online: https://doi.org/10.1063/1.4942603

View Table of Contents: http://aip.scitation.org/toc/apl/108/8

Published by the American Institute of Physics

\section{Articles you may be interested in}

Edge-enhanced Raman scattering in narrow sGe fin field-effect transistor channels

Applied Physics Letters 106, 033107 (2015); 10.1063/1.4906537

Edge-enhanced Raman scattering in Si nanostripes

Applied Physics Letters 94, 131907 (2009); 10.1063/1.3110964

Relation between Raman frequency and triaxial stress in Si for surface and cross-sectional experiments in microelectronics components

Journal of Applied Physics 118, 053101 (2015); 10.1063/1.4927133

Stress measurements in silicon devices through Raman spectroscopy: Bridging the gap between theory and experiment

Journal of Applied Physics 79, 7148 (1998); 10.1063/1.361485

Optical functions of silicon at elevated temperatures

Journal of Applied Physics 76, 3758 (1998); 10.1063/1.357378

Characterization of anisotropic stress around $\mathrm{Si}$ trenches by polarized Raman spectroscopy

Journal of Applied Physics 78, 941 (1998); 10.1063/1.360287

\section{Scilight}

Sharp, quick summaries illuminating the latest physics research

\section{Sign up for FREE!}

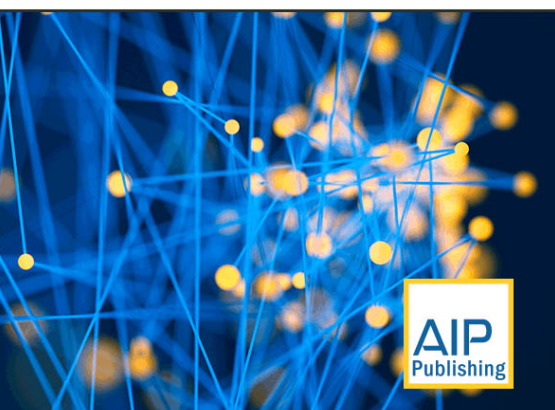




\title{
Nanofocusing of light into semiconducting fin photonic crystals
}

\author{
J. Bogdanowicz, ${ }^{1, a)}$ T. Nuytten, ${ }^{1}$ A. Gawlik, ${ }^{1,2,3}$ A. Schulze, ${ }^{1}$ I. De Wolf, ${ }^{1,4}$ \\ and W. Vandervorst ${ }^{1,2}$ \\ ${ }^{1}$ IMEC, Kapeldreef 75, B-3000 Leuven, Belgium \\ ${ }^{2}$ Instituut voor Kern-en Stralingsfysika, KU Leuven, Celestijnenlaan 200D, B-3001 Leuven, Belgium \\ ${ }^{3}$ Laboratory for Optical Spectroscopy of Nanostructures, Department of Experimental Physics, Wroclaw \\ University of Technology, Wybrzeze Wyspianskiego 27, 50-370 Wroclaw, Poland \\ ${ }^{4}$ Department of Material Science, KU Leuven, B-3001 Leuven, Belgium
}

(Received 7 December 2015; accepted 10 February 2016; published online 23 February 2016)

\begin{abstract}
This letter demonstrates experimentally and investigates theoretically the possibility for enhanced light coupling into periodic arrays of nanoscale semiconducting fins. Using Raman spectroscopy, we show that an electromagnetic field impinging upon such periodic structures can be confined into the semiconducting regions when the ratio $\mathrm{W} / \lambda_{0}$ of the fin width to the incident wavelength is sufficiently small and when the incident light polarization is parallel to the fin edges. As we demonstrate based on band structure calculations and finite-element simulations, this corresponds to the availability and excitation of a dielectric-band mode of the constituted photonic crystal waveguide, i.e., a mode guided inside the semiconducting fins. The understanding of this nanofocusing behavior opens the way to a plethora of applications including the optical metrology of deepsubwavelength non-planar semiconductor devices. (C) 2016 AIP Publishing LLC.
\end{abstract}

[http://dx.doi.org/10.1063/1.4942603]

The very high sensitivity of Raman spectroscopy to local light intensity makes it an ideal technique for probing optical resonance, such as widely employed using metals. ${ }^{1-4}$ Recently, Raman measurements on periodic arrays of deepsubwavelength semiconducting trenches have also put forward the existence of a resonant coupling phenomenon when the light polarization is aligned with the trench edges and the trench width becomes small compared to the incident wavelength. ${ }^{5,6}$ The discovery of this important effect in nanoscale semiconductor fins has relaunched the hope for the possible non-contact characterization of mechanical stress in state-ofthe-art semiconductor devices with Raman spectroscopy. ${ }^{7-9}$ Since the advent of the three-dimensional devices embodied by the so-called finFETs (fin field-effect transistors), Raman measurements on such devices have indeed become increasingly challenging, given their small dimensions compared to the probing laser beam and their complex architecture. Initially, this phenomenon has been attributed to enhanced light transmission via the fin edges but, though this heuristic satisfactorily explains the enhancement in some cases, it fails to predict the actual conditions required to trigger the resonance when varying the light polarization or the fin width. ${ }^{6}$ In this letter, we show that this resonance is actually caused by the excitation of a propagating mode in the semiconducting fins, i.e., a mode of the so-called dielectric bands of the one-dimensional photonic crystal created by the fins. This letter starts by demonstrating experimentally that this phenomenon is linked to the excitation of a propagating mode by investigating Raman scattering on dedicated arrays of Ge fins embedded in $\mathrm{SiO}_{2}$. Further, assuming the studied arrays are one-dimensional photonic crystals, we derive their band structure, i.e., the dispersion relation of the modes which can propagate inside this structure. These calculations are

a)Janusz.Bogdanowicz@imec.be corroborated by finite-element simulations and demonstrate the existence of a so-called nanofocusing mode guided inside the semiconducting fins but behaving similarly to light propagating inside the surrounding dielectric.

Raman spectroscopy is mostly used in semiconductor devices to measure composition, stress, crystallinity, and in some cases even temperature or doping level. One of less well known strengths of Raman spectroscopy is its ability to probe and discriminate light propagation inside different materials illuminated by the same light source with a very high sensitivity. This is extremely useful when it comes to understanding light coupling into complex heterostructures. Fig. 1 shows Raman spectroscopy results measured on periodic arrays of narrow $\mathrm{Ge} / \mathrm{Si}_{0.3} \mathrm{Ge}_{0.7} / \mathrm{Si}$ fins embedded in $\mathrm{SiO}_{2}$ (Fig. 1, right). These arrays have been fabricated using a
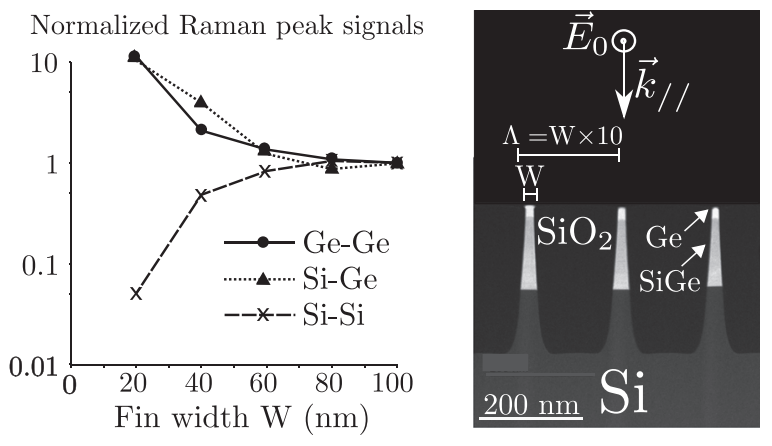

FIG. 1. Left: Normalized intensities of the Ge-Ge (full line), Si-Ge (dotted line), and $\mathrm{Si}-\mathrm{Si}$ (interrupted line) Raman peaks measured with an excitation wavelength of $532 \mathrm{~nm}$ with electric field polarized parallel to the fin edges, as a function of the width of the embedded $\mathrm{Ge} / \mathrm{Si}_{.3} \mathrm{Ge}_{.7} / \mathrm{Si}$ fins shown on the right. The fin coverage is kept at a constant $10 \%$ over the different structures, i.e., the pitch $\Lambda \sim \mathrm{W} \times 10$. Right: Transmission electron micrograph of three fins belonging to an array of $\mathrm{Ge}(30 \mathrm{~nm}) / \mathrm{Si}_{.3} \mathrm{Ge}_{.7}(130 \mathrm{~nm}) /$ $\mathrm{Si}(125 \mathrm{~nm})$ fins embedded in $\mathrm{SiO}_{2}$ with pitch $\Lambda=200 \mathrm{~nm}$ and fin width $\mathrm{W}=20 \mathrm{~nm}$. The length of the fins in the direction perpendicular to the figure is $10 \mu \mathrm{m}$. 
fin-replacement process. ${ }^{10}$ More specifically, Fig. 1 shows the dependence on fin width of the normalized intensities of the Ge-Ge, Si-Ge, and Si-Si Raman peak signals for an incident laser light of wavelength $\lambda_{0}=532 \mathrm{~nm}$ polarized parallel to the fins edges. More information about the measurement conditions can be found in Ref. 6. Two critical observations can be made from this figure. First, a strong enhancement with decreasing fin width is observed for both the Ge-Ge signal coming from the surface and the Si-Ge signal coming from deeper inside the structure. Though this enhancement is most dramatic for a $532 \mathrm{~nm}$ incident wavelength, similar but much less pronounced behavior has also been observed at 405 and $633 \mathrm{~nm}$ (not shown). Second, just as the Ge-Ge and $\mathrm{Si}-\mathrm{Ge}$ signals rise, a simultaneous drop in the $\mathrm{Si}-\mathrm{Si}$ signal is observed. It is important to stress that, while the fin width $\mathrm{W}$ changes in the measured structures of Fig. 1, the pitch $\Lambda$ also changes in order to maintain a constant fin coverage $\mathrm{W} / \Lambda=0.1$. The observed enhancement therefore cannot be attributed to just an increase in the probed $\mathrm{Ge}$ or SiGe volumes (beam width $\sim 1-1.5 \mu \mathrm{m}$ ). As mentioned above, the $\mathrm{Ge}-\mathrm{Ge}$ and $\mathrm{Si}-\mathrm{Ge}$ signals can be used as indicators of the light intensity coupled, respectively, at the surface of the Ge fin and $10-100 \mathrm{~nm}$ below this surface. Similarly, the Si-Si signal can be used to evaluate the light intensity which has propagated deeper down to the Si substrate. In the Raman data of Fig. 1, the enhancement of the Ge-Ge signal therefore points toward the strong focusing of the light inside the nanoscale Ge fins when the fin width decreases. This interpretation also agrees with the strong decay in $\mathrm{Si}-\mathrm{Si}$ signal since very large absorption is expected in the Ge fin, which would prevent light from reaching the Si substrate. Finally, the enhancement of the Si-Ge signal shows that light is not only coupled into the Ge fins but also propagates down to the SiGe region of the fin. In summary, the experimental data of Fig. 1 clearly indicate the intense excitation of a so-called nanofocusing mode, i.e., a guided mode propagating inside the Ge fins, when the fin width is sufficiently small.

In order to prove and understand theoretically the existence of this nanofocusing mode, one has to realize that the periodic array of Fig. 1 (right) constitutes, in first approximation, a photonic crystal waveguide of successive Ge and $\mathrm{SiO}_{2}$ layers. ${ }^{11}$ The peculiar propagation of light inside such waveguides can then be studied analytically based on their band structure, i.e., the dispersion relations of the possible guided modes inside the structure. Fig. 2 shows the band structure derived using the plane wave expansion method $^{12-14}$ for the case closest to the one considered in this paper, i.e., an infinite one-dimensional $\mathrm{Ge} / \mathrm{SiO}_{2}$ photonic crystal with pitch $\Lambda$ and a Ge fin width $\mathrm{W}=\Lambda / 10$, inside which propagate the transverse-electric (TE) modes, i.e., modes with an electric field polarized parallel to the fin edges (Fig. 2, right). In essence, it is the fundamental mode highlighted in red in Fig. 2 which accounts for the nanofocusing effect put forward in Fig. 1. More specifically, it is the combination of two features of this fundamental mode which triggers the enhanced coupling into the fins. First, this mode is located between the $\mathrm{SiO}_{2}$ and Ge light lines (respectively, interrupted and dotted black lines), i.e., on a dielectric band, and is therefore confined in the Ge fins. ${ }^{11}$ Furthermore, for small fin widths, this band remains relatively close to the

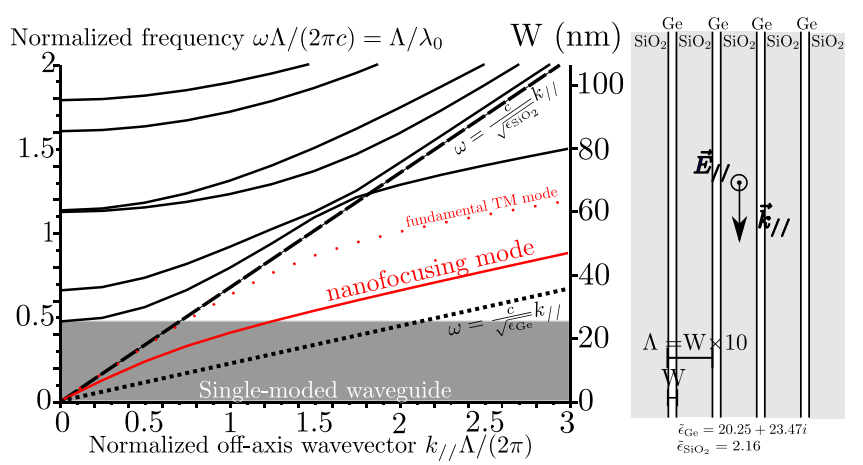

FIG. 2. Band structure for the electromagnetic modes propagating off axis, i.e., along the fins with a wavevector $\mathrm{k}_{/ /}$, with the electric field polarized along the fins (TE polarization) inside the photonic crystal displayed on the right, i.e., a periodic array of $\mathrm{Ge}$ fins of width $\mathrm{W}$ embedded in $\mathrm{SiO}_{2}$ with a pitch $\Lambda=\mathrm{W} \times 10$. The right vertical axis shows the fin width values for $\lambda_{0}=532 \mathrm{~nm}$ and $\mathrm{W}=\Lambda / 10$. The fundamental nanofocusing mode (solid red line) located between the $\mathrm{SiO}_{2}$ and Ge light lines (respectively, thick interrupted and dotted lines) explains the enhanced light coupling into the narrowest fins. For comparison, the red dotted line shows the fundamental transverse-magnetic (TM) mode, which follows the $\mathrm{SiO}_{2}$ light line and hence does not allow nanofocusing. The used values of the dielectric constants can be found in Ref. 18 .

$\mathrm{SiO}_{2}$ light line, i.e., although the nanofocusing mode propagates in the Ge fin, it behaves similarly to light propagating inside bulk $\mathrm{SiO}_{2}$ and hence is easy to excite (effective dielectric constant of the complete system $\left.\sim \varepsilon_{\mathrm{SiO}_{2}}\right)$. Second, as highlighted by the shaded area in Fig. 2, the fundamental nanofocusing mode is the only propagating mode of the waveguide when the normalized frequency (left vertical axis) is less than approximately 0.5 , i.e., when $\mathrm{W}<26 \mathrm{~nm}$ (right vertical axis). In other words, in the case of small fin widths belonging to the shaded area, light propagation in $\mathrm{SiO}_{2}$ is forbidden. The only option for TE light to couple into the fin array is to excite the nanofocusing mode which propagates inside the Ge fins. As the fin width is increased beyond the shaded area, on the other hand, modes above the $\mathrm{SiO}_{2}$ light line start appearing and light propagation inside the $\mathrm{SiO}_{2}$ is allowed. Simultaneously, the nanofocusing mode band moves further away from the $\mathrm{SiO}_{2}$ light line and, as a result, becomes more difficult to excite. Altogether, the light intensity coupled into the semiconducting fins decreases, in agreement with Fig. 1. Note that another dielectric band mode also exists for fin width around $60 \mathrm{~nm}$. It, however, is expected to have much less impact on the light coupling since it always coexists with modes propagating inside $\mathrm{SiO}_{2}$.

To confirm our theoretical understanding of the nanofocusing phenomenon, Fig. 3 shows the simulated amplitude of the electric fields $\left|\mathrm{E}_{\mathrm{Ge}}\right|$ and $\left|\mathrm{E}_{\mathrm{Si}}\right|$, respectively, in the center of the air/Ge and $\mathrm{SiO}_{2} / \mathrm{Si}$ interfaces as obtained with finiteelement modeling (FEM) calculations using COMSOL. ${ }^{15}$ These calculations assume a uniform plane wave with $\lambda_{0}=532 \mathrm{~nm}$ normally incident on an array of $\mathrm{Ge} / \mathrm{Si}_{.3} \mathrm{Ge}_{.7} / \mathrm{Si}$ fins with a fin width varying from 0 to $100 \mathrm{~nm}$ and a pitch $\Lambda=\mathrm{W} \times 10$ (see geometry in Fig. 3(a), right). The simulations are performed assuming one single fin embedded in $\mathrm{SiO}_{2}$ with periodic boundary conditions, which is equivalent to simulating the collective behavior of an infinite number of fins. Concerning the fin geometry, vertical sidewalls like in the band structure calculations of Fig. 2 are assumed rather 

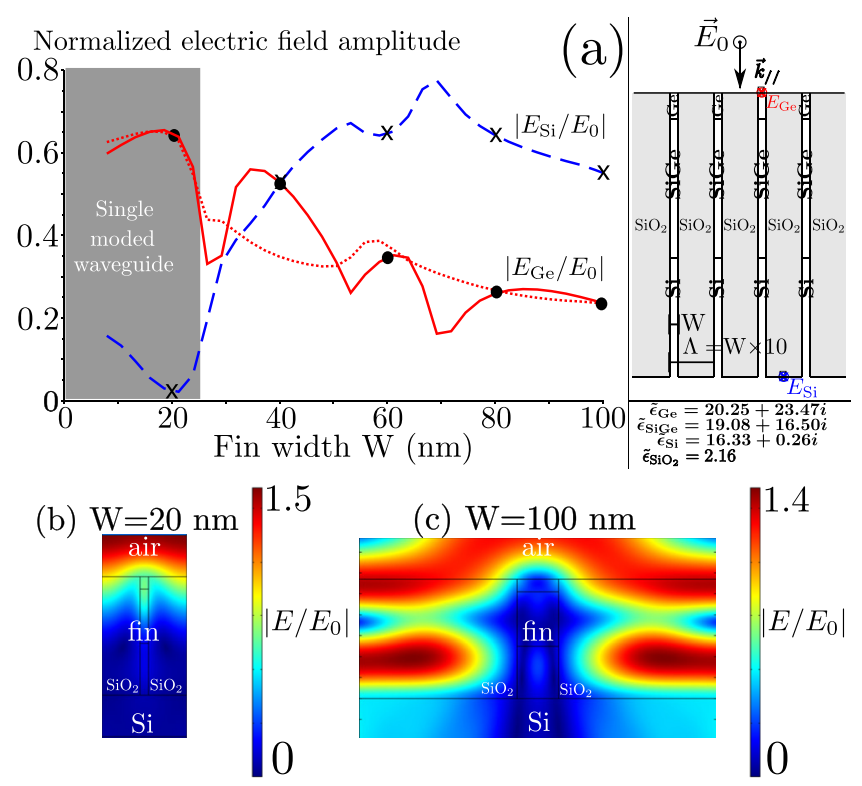

FIG. 3. (a) (Left) FEM simulations of the normalized amplitude of the electric fields $\left|\mathrm{E}_{\mathrm{Ge}}\right|$ (solid red line) and $\left|\mathrm{E}_{\mathrm{Si}}\right|$ (interrupted blue line), respectively, in the center of the air/Ge interface and of the $\mathrm{SiO}_{2} / \mathrm{Si}$ interface as a function of the pitch of the fin array shown on the right. The dotted red line gives $\left|\mathrm{E}_{\mathrm{Ge}}\right|$ in the case of infinitely deep fins. A normally incident plane wave of wavelength $\lambda_{0}=532 \mathrm{~nm}$ and amplitude $\left|\mathrm{E}_{0}\right|$ polarized parallel to the fin edges is assumed. The used values of the dielectric constants of $\mathrm{Si}$ and $\mathrm{Ge}$ can be found in Ref. 18 and the value for $\mathrm{Si}_{3} \mathrm{Ge}_{7}$ has been obtained by interpolation. The symbols indicate the pitch values of the measurements of Fig. 1. (Right) Four of the $\mathrm{Ge}(30 \mathrm{~nm}) / \mathrm{Si}_{3} \mathrm{Ge}_{7}(130 \mathrm{~nm}) /$ $\mathrm{Si}(125 \mathrm{~nm})$ fins of the simulated two-dimensional array. (b) Distribution of the normalized electric field inside the unit cell of a nanofocusing array of 20-nm wide fins. (c) Distribution of the normalized electric field inside the unit cell of an array of 100-nm wide fins.

than the actual tapered geometry of the fins of Fig. 1 (right). When coupling into tapered waveguides, light indeed excites the local modes characterized by the local width of the waveguide. ${ }^{16}$ It is therefore expected and easy to confirm numerically that the tapered geometry of the real fins only introduces a small perturbation of the nanofocusing effect. Looking at Fig. 3(a), one can note the very good agreement between the simulations and the experimental data of Fig. 1. The symbols in Fig. 3 indeed highlight the simulated electric field values at the fin widths where the Raman measurements of Fig. 1 were performed and reflect a similar decrease (respectively, increase) in the electric field in the Ge fin (respectively, the Si substrate). Furthermore, these simulations also confirm the interpretation of the band structure given above. Most strikingly, the two regions of Fig. 2 are readily recognized in Fig. 3(a). First, the shaded area of Fig. 3(a) where very high $\left|E_{G e}\right|$ and very low $\left|\mathrm{E}_{\mathrm{Si}}\right|$ are obtained perfectly corresponds to the shaded area of Fig. 2 where only the nanofocusing mode can be excited. In that region, $\left|\mathrm{E}_{\mathrm{Ge}}\right|$ is close to the light amplitude transmitted into bulk $\mathrm{SiO}_{2}(\sim 0.8)$, i.e., the nanofocusing mode behaves roughly like light propagating in bulk $\mathrm{SiO}_{2}$, as was inferred from the band structure as well. To better illustrate the nanofocusing effect present in this region, Fig. 3(b) shows the distribution of the electric field inside the unit cell of an array of 20-nm wide fins. The propagation of the nanofocusing mode can be observed as it confines the incident light into the fins. In the Ge fins investigated in this paper, the nanofocusing mode only propagates down to a few tens of nanometers deep due to the high absorption coefficients of $\mathrm{Ge}$ and $\mathrm{SiGe}$, but it would propagate much deeper in a fin made of a less absorptive medium. Second, as the pitch is increased beyond the shaded area, $\left|\mathrm{E}_{\mathrm{Ge}}\right|$ very quickly drops while $\left|\mathrm{E}_{\mathrm{Si}}\right|$ rises, as expected from the appearance in Fig. 2 of further modes becoming available for light propagation inside the non-absorbing oxide. This situation is illustrated in Fig. 3(c) showing the electric field distribution in an array of 100-nm wide fins, where it can be clearly observed that most of the light propagates inside the oxide. In spite of the excellent agreement between Figs. 2 and 3, multiple oscillations on top of the decaying envelope of $\left|\mathrm{E}_{\mathrm{Ge}}\right|$ with minima around 25, 50, and $75 \mathrm{~nm}$ fin width are observed, which cannot be explained based on the band structure of Fig. 2. These oscillations are actually to be attributed to Fabry-Perot resonances in the oxide as a result of the finiteness of the fin height in Fig. 3. To prove this statement, Fig. 3(a) also shows the expected behavior of $\left|\mathrm{E}_{\mathrm{Ge}}\right|$ in case of an infinite Ge fin height (dotted red line), where most oscillations have indeed disappeared. The infinite fin height is simulated by assuming a $5-\mu \mathrm{m}$ thick oxide layer with a small imaginary refractive index $\left(\mathrm{k}_{\mathrm{SiO} 2}=0.01\right)$, which prevents any light from reaching the $\mathrm{SiO}_{2} / \mathrm{Si}$ interface. Note that, interestingly, a slight increase in $\left|\mathrm{E}_{\mathrm{Ge}}\right|$ is still visible around $60 \mathrm{~nm}$ fin width, but this is in agreement with the existence of an extra dielectric band mode (Fig. 2).

As a final remark, we would like to make use of the understanding developed in this paper to give some general guidance on the expected conditions required for enhanced coupling into the fins. As shown above, the basic condition for the existence of a nanofocusing mode is that its band should be located between the $\mathrm{SiO}_{2}$ and Ge light lines, which requires the fin width to be only a fraction of the incident wavelength. However, three other parameters can also impact the nanofocusing phenomenon. First, two parameters can change the band structure, i.e., the light polarization and the fin coverage. Furthermore, also the fin height can impact the field inside the fins in case of lowly absorptive materials, though it does not alter the band structure. Let us look separately at how to maximize the enhancement based on variations in these three parameters. First, concerning the polarization of the incident light, the enhanced coupling strictly requires that it should be parallel to the fins. ${ }^{5,6}$ The band structure for modes polarized perpendicular to the fins shows a fundamental mode following the $\mathrm{SiO}_{2}$ light line when the waveguide is single-moded (red dotted line of Fig. 2); the nanofocusing effect is therefore not allowed for perpendicular polarization. Second, the fin coverage should remain rather limited. As the pitch decreases at a constant fin width, the nanofocusing mode indeed quickly moves closer to the $\mathrm{Ge}$ light line and hence becomes more difficult to excite. In the case of the Ge fins of this paper, a $30-40 \%$ coverage is already expected to significantly degrade the coupling. At $50 \%$ coverage, the effective dielectric constant $\sim \varepsilon_{\mathrm{Ge}}$ and the mode couples into the fin as it would transmit into bulk Ge; the enhancement is lost. It is interesting to note that there is, however, no strict lower limit to the fin coverage. In the case of a single illuminated fin, i.e., for an infinite pitch, the $\mathrm{TE}_{0}$ mode of the constituted $\mathrm{SiO}_{2} / \mathrm{Ge} / \mathrm{SiO}_{2}$ (dielectric) waveguide indeed 
takes the role of the nanofocusing mode ${ }^{16}$ and the field enhancement inside the fin is preserved. Third, the fin height should also be chosen with care in case of lowly absorptive fin materials. Although the latter parameter has no impact on the nanofocusing mode itself, Fabry-Perot interferences may indeed dramatically increase or reduce the electric field amplitude inside the fins if the light coupled into the fins can bounce back and forth at the air/fin surface and the fin/substrate interface. Provided the substrate is less refractive than the fin material, the general rule for constructive interference of these reflections inside the fins is that the fin height should be an odd multiple of $\pi / k_{/ /}{ }^{17}$

To conclude, this letter demonstrates the existence of an enhancement allowing the intense focusing of light into nanoscale semiconducting fin arrays. Based on a combination of Raman spectroscopy measurements and photonic crystal waveguide theory, we have shown that the enhancement is due to the existence of a nanofocusing mode confined in the semiconducting fins when the incident light is polarized parallel to the fin edges and the fin width is only a small fraction of the incident wavelength. The existence and understanding of this enhancement is the key towards extending nondestructive optical measurements of semiconductor devices to dimensions far beyond the diffraction limit and creating hence the sensitivity required to probe very small devices.

The authors are indebted to R. Loo, the EPI group, and the Logic Program of imec for the growth of the fin arrays.
${ }^{1}$ R. Zhang, Y. Zhang, Z. C. Dong, S. Jiang, C. Zhang, L. G. Chen, L. Zhang, Y. Liao, J. Aizpurua, Y. Luo, J. L. Yang, and J. G. Hou, Nature 498, 82-86 (2013).

${ }^{2}$ J. Steidtner and B. Pettinger, Phys. Rev. Lett. 100, 236101 (2008).

${ }^{3}$ H. Hashiguchi, M. Takei, D. Kosemura, and A. Ogura, Appl. Phys. Lett. 101, 172101 (2012).

${ }^{4}$ G. M. Vanacore, M. Chaigneau, N. Barrett, M. Bollani, F. Boioli, M. Salvalaglio, F. Montalenti, N. Manini, L. Caramella, P. Biagioni, D. Chrastina, G. Isella, O. Renault, M. Zani, R. Sordan, G. Onida, R. Ossikovski, H.-J. Drouhin, and A. Tagliaferri, Phys. Rev. B 88, 115309 (2013).

${ }^{5}$ V. Poborchii, T. Tada, and T. Kanayama, Appl. Phys. Lett. 94, 131907 (2009).

${ }^{6}$ T. Nuytten, T. Hantschel, D. Kosemura, A. Schulze, I. D. Wolf, and W. Vandervorst, Appl. Phys. Lett. 106, 033107 (2015).

${ }^{7}$ I. D. Wolf, Semicond. Sci. Technol. 11, 139 (1996).

${ }^{8}$ I. D. Wolf, H. E. Maes, and S. K. Jones, J. Appl. Phys. 79, 7148 (1996).

${ }^{9} \mathrm{~W}$. Vandervorst, in Proceedings of the International Conference on the Frontiers of Characterization and Metrology for Nanoelectronics, 2015.

${ }^{10}$ R. Loo, J. Sun, L. Witters, A. Hikavyy, B. Vincent, Y. Shimura, P. Favia, O. Richard, H. Bender, W. Vandervorst, N. Collaert, and A. Thean, in Proceedings of the Silicon-Germanium Technology and Device Meeting (ISTDM) (2014), p. 19.

${ }^{11}$ J. D. Joannopoulos, S. G. Johnson, J. N. Winn, and R. D. Meade, Photonic Crystals: Molding the Flow of Light, 2nd ed. (Princeton University Press, 2008).

${ }^{12}$ Z. Zhang and S. Satpathy, Phys. Rev. Lett. 65, 2650-2653 (1990).

${ }^{13}$ K. M. Ho, C. T. Chan, and C. M. Soukoulis, Phys. Rev. Lett. 65, 3152-3155(1990).

${ }^{14}$ J. D. Shumpert, Ph.D. thesis, University of Michigan, 2001.

${ }^{15}$ See www.comsol.com/comsol-multiphysics for frequency domain electromagnetic wave modeling.

${ }^{16}$ A. W. Snyder and J. D. Love, Optical Waveguide Theory (Chapman and Hall, 1983).

${ }^{17}$ M. Born and E. Wolf, Principles of Optics, 6th ed. (Pergamon Press, 1999).

${ }^{18}$ D. E. Aspnes and A. A. Studna, Phys. Rev. B 27, 985 (1983). 